\title{
Design and performance studies of the calorimeter system for an FCC-hh experiment
}

\author{
C. Helsens, on behalf of the FCC-hh detector working group \\ CERN, CH-1211 Geneva 23, Switzerland
}

\section{A R T I C L E I N F O}

\section{Keywords:}

Calorimeters

Noble liquid detectors (scintillation, ionisation, double-phase)

Scintillation and light emission processes (solid

Gas and liquid scintillators)

\begin{abstract}
A B S T R A C T
The physics reach and feasibility of the Future Circular Collider are currently being investigated in the form of a Conceptual Design Report. The ultimate goal of the FCC is to collide protons with a centre-of-mass energies of $100 \mathrm{TeV}$, thus extending the reach of the current HEP facilities. This high-energy regime opens new opportunities for the discovery of physics beyond the standard model, but also new constraints on the detector design. As at $100 \mathrm{TeV}$ a large fraction of the $\mathrm{W}, \mathrm{Z}, \mathrm{H}$ bosons and top quarks are produced with a significant boost, it implies an efficient reconstruction of high energetic objects. The reconstruction of those boosted objects sets the calorimeter performance requirements in terms of energy resolution, containment of highly energetic hadron showers, and high transverse granularity. The detectors designed for the FCC experiments need to tackle harsh conditions of the unprecedented collision energy and instantaneous luminosity. They also must be able to deal with a very high number of collisions per bunch crossings (pile-up). Excellent energy and angular resolution, also for low energetic particles, are therefore needed in order to meet the demands based on the physics benchmarks like Higgs self-couplings. In this proceedings are presented the current baseline technologies for the calorimeter system of the FCC-hh reference detector and present first results of the performance studies with the combined calorimeters, meeting the energy resolution goal.
\end{abstract}

Contents

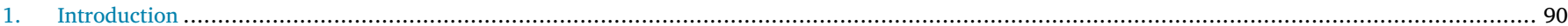

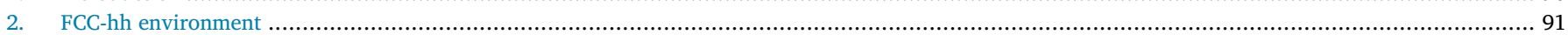

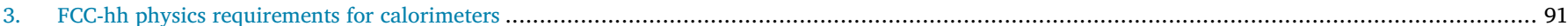

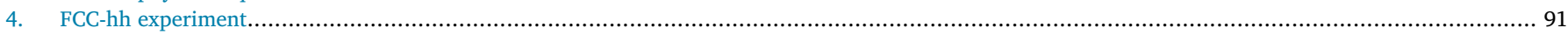

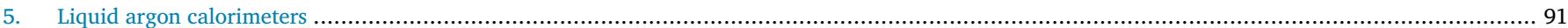

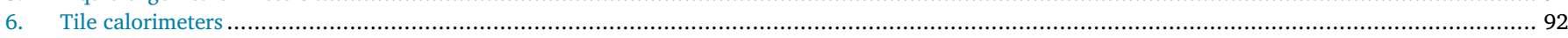

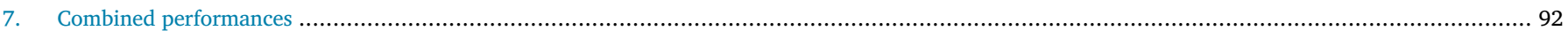

8. Summary

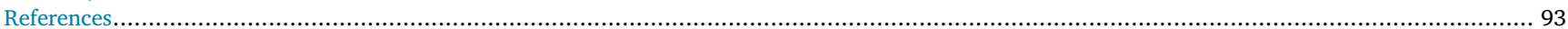

\section{Introduction}

The Future Circular Collider (FCC) [1] project is an ambitious plan for a post-LHC era within CERN complex. It includes studies of a pp circular collider with a centre-of-mass energy of $100 \mathrm{TeV}$ (FCC-hh), which defines the infrastructure. An electron-positron collider (FCC-ee) is considered as a possible first step using the same tunnel as the pp collider. Moreover, the option of an electron-proton collider is investigated (FCC-he). Given the topology of the CERN area, the maximum tunnel circumference that can be reached is almost $100 \mathrm{~km}$ [2]. The centre-of-mass energy is then defined by the maximal bending power of the dipole magnets. For this study it is commonly assumed that
$16 \mathrm{~T}$ magnets can be built at large scale, thus $100 \mathrm{TeV}$ centre-ofmass energy of pp collisions can be targeted. The most recent running scenario under consideration is 10 years with a peak luminosity of $5 \times 10^{34} \mathrm{~cm}^{-2} \mathrm{~s}^{-1}$ (so-called baseline scenario), followed by 15 years running with $30 \times 10^{34} \mathrm{~cm}^{-2} \mathrm{~s}^{-1}$ (ultimate scenario). This will result in $\mathcal{O}(20) \mathrm{ab}^{-1}$ integrated luminosity per experiment. The outcome of the design studies is being summarised in the Conceptual Design Report, to be submitted for the European Strategy Update in 2019. This paper concentrates on the electromagnetic (ECal) and hadronic (HCal) calorimeters design and performances for the FCC-hh machine, with the focus on the interplay between the physics benchmarks driving

E-mail address: clement.helsens@cern.ch. 
the detector design. The studies presented in this proceedings are all performed within the FCC software framework, FCCSW [3].

\section{FCC-hh environment}

Compared to LHC, the total inelastic cross-section only increases by a factor of 1.25 . Hence the radiation level is mostly driven by the instantaneous luminosity which is a factor 100 larger than LHC. It has implication on the radiation levels for the calorimeters as the $1 \mathrm{MeV}$ neutron equivalence fluence would raise up to $4 \times 10^{15(14)} \mathrm{cm}^{-2}$ in the barrel for the ECal (HCal) and up to $2 \times 10^{16} \mathrm{~cm}^{-2}$ in the end-caps. Assuming $25 \mathrm{~ns}$ bunch spacing, the average number of pile-up collisions, $\langle\mu\rangle$, is as high as 1000 in the ultimate scenario. Mitigation of this high pile-up may be the most important challenge for the detectors and the reconstruction methods on the FCC-hh.

\section{FCC-hh physics requirements for calorimeters}

Extending the energy regime to $100 \mathrm{TeV}$ leads to a natural gain in the exploratory potential of a collider. As an example, direct production of heavy resonances can be probed up to masses of $40 \mathrm{TeV}$ and supersymmetric top-partners up to $10 \mathrm{TeV}$. In addition to such heavy states, FCC-hh has a large programme of precision physics, complementary to an $e^{+} e^{-}$collider. It includes Higgs self-coupling (reach $\Delta \lambda / \lambda \approx$ $4 \%)$, Higgs rare decays $(\mu \mu, Z \gamma)$ and electro-weak/top-physics in new extreme dynamical regimes. The physics potential has already been extensively studied and is described in [4].

Low $p_{T}$ physics produced at threshold (electro-weak, Higgs, top) is more forward. Hence larger $|\eta|$ coverage (up to 6) compared to LHC is needed. For precision physics, like $H H \rightarrow b \bar{b} \gamma \gamma$, excellent energy and angular resolution at low energy is required. In addition, small noise and stochastic terms of energy resolution, robustness versus pileup and $\pi^{0}$ rejection capabilities are key elements of the design. Excellent lateral and longitudinal granularities are required to make particle-flow algorithm more effective and to achieve pointing capabilities needed to trigger on a light state like $H H \rightarrow b \bar{b} \gamma \gamma$. It will also be very important to help with pile-up rejection.

In terms of detector design, sensitivity to coloured hadronic resonances up to $40 \mathrm{TeV}$, implies full containment for jets with $p_{T} \sim$ $20 \mathrm{TeV}$ hence a small constant term of energy resolution. In addition, the containment limits the punch through and helps with the muon identification. Correct assessment of the calorimeter containment drives detector size thus the magnet dimension which is the most expensive element of such detector. The FCC-hh also has sensitivity for boosted resonances (ex: $Z^{\prime} \rightarrow t \bar{t}$ or $G \rightarrow W^{+} W^{-}$) up to masses of $20 \mathrm{TeV}$. In such scenarios called boosted objects, a $W$ jet with $p_{T}=10 \mathrm{TeV}$ sees its decay product separated by $\Delta R \sim 0.02$ which correspond to the typical ECal cell size. Very high granularity is needed to resolve such substructure. But questions that need to be addressed in more details later in the process are: has calorimetry alone the capability to resolve such objects? High granularity being costly, does it translate to actual separation power?

\section{FCC-hh experiment}

The reference layout of the FCC-hh detector is presented in Fig. 1 and discussed at large during the last FCC week [5]. The design of the detector is inspired by the current running experiments at the LHC. The electromagnetic calorimeters, indicated in dark blue, use liquid argon (LAr) technology with lead absorbers up to $|\eta|<2.5$ and LAr technology with copper absorbers in the forward region $(2.3<|\eta|<6)$. The hadronic calorimeter, indicated in green and light blue, is composed of scintillating tiles and a mixture of stainless steel and lead in the central region $(|\eta|<1.8)$. LAr technology with copper absorbers in the end-caps $(1.6<|\eta|<2.5)$ and in the forward region $(|\eta|<6)$ is used. Such technology choices have been made due to radiation hardness requirements. The barrel calorimeters are placed inside a solenoid producing a magnetic field of $4 \mathrm{~T}$. The overall length of the FCC-hh detector is approximately $50 \mathrm{~m}$ with a radius of $10 \mathrm{~m}$ which is very similar to the dimensions of the ATLAS experiment [6] at the LHC.

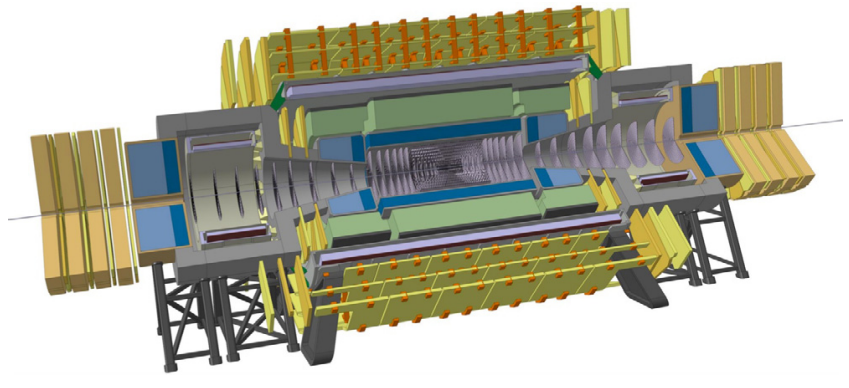

Fig. 1. A reference design of the FCC-hh experiment [5]. (For interpretation of the references to colour in this figure legend, the reader is referred to the web version of this article.)

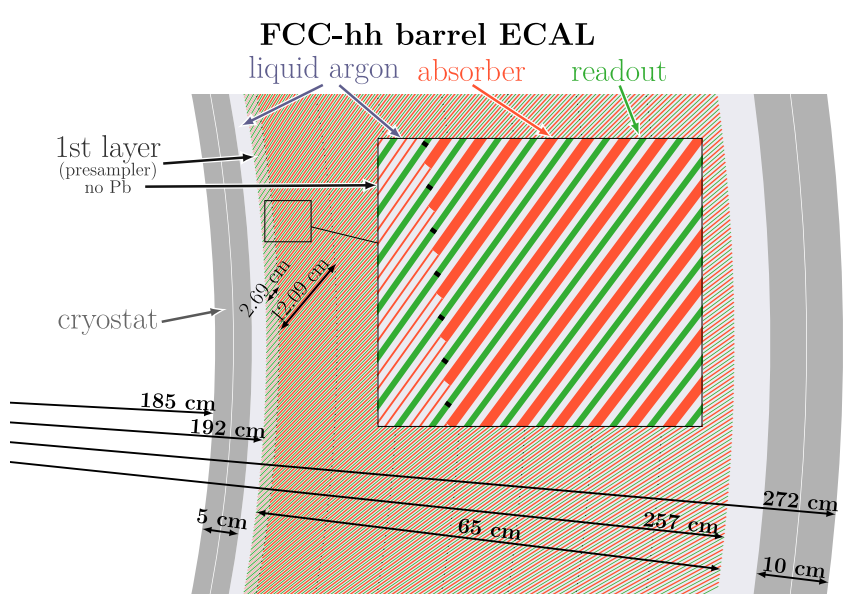

Fig. 2. The cross section of the electromagnetic barrel calorimeter.

\section{Liquid argon calorimeters}

Increasing the longitudinal segmentation of ATLAS like [7] barrel electromagnetic calorimeter is not possible in its current configuration. Using straight multilayer electrodes allows to easily read out all the longitudinal layers and it has also the advantage of being easier to construct while keeping a very high accuracy. Overall, the FCC design is ten times more granular than ATLAS calorimeter. For the barrel part, as shown in Fig. 2, two millimetres thick absorbers are inclined by $50^{\circ}$ angle, leading to an increase of the LAr gap with depth from 1.15 to $3.09 \mathrm{~mm}$. This changing sampling fraction with calorimeter depth is taken into account in the calibration. The number of longitudinal layers is set to 8 with the first one without lead to serve as a pre-sampler. The achieved granularity is $\Delta \eta \times \Delta \phi=0.01 \times 0.009(\Delta \eta=0.0025$ in 2nd layer). For the end-caps both ECal and HCal are within same cryostat. The ECal is composed of $1.5 \mathrm{~mm}$ lead discs with $0.5 \mathrm{~mm}$ LAr gap in between, the first layer to act as a pre-sampler. For the HCal, $2 \mathrm{~cm}$ copper discs with a $2 \mathrm{~mm}$ LAr gap are used. The forward calorimeters are simulated with same layout using $0.1 \mathrm{~mm}$ LAr gap and 1 (4) $\mathrm{cm}$ copper discs in ECal (HCal).

Simulation of single electrons without noise in the detector and reconstructed using the sliding window algorithm [8] (with a size of $\Delta \eta \times \Delta \phi=0.07 \times 0.17$ ), shows excellent performance over the acceptance range. For example in the barrel an energy resolution of $\sigma_{E} / E=$ $8.1 \% \sqrt{E} \oplus 0.2 \%$ is achieved. The electronics noise is estimated for PCB readout and is approximately $300 \mathrm{MeV}$ in a cluster. The in-time pileup noise is estimated from the simulation of minimum-bias events and could be as high as $6 \mathrm{GeV}$ per cluster for $\langle\mu\rangle=1000$ at $|\eta|=1.4$ for a non-optimised reconstruction with a fixed cluster size. It is also possible to reject the energy deposited from pile-up vertices tagged by 


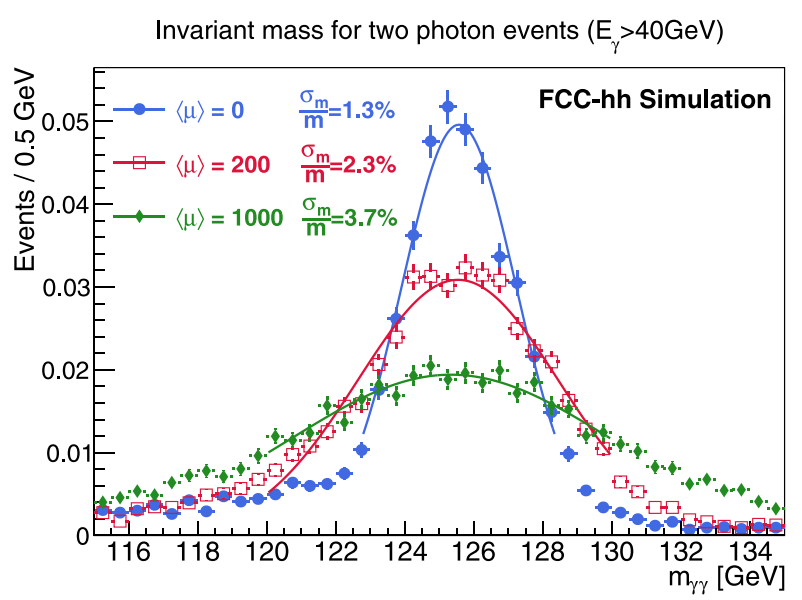

Fig. 3. Di-photon invariant mass distribution for a $125 \mathrm{GeV}$ Higgs boson in different pile-up scenarios.

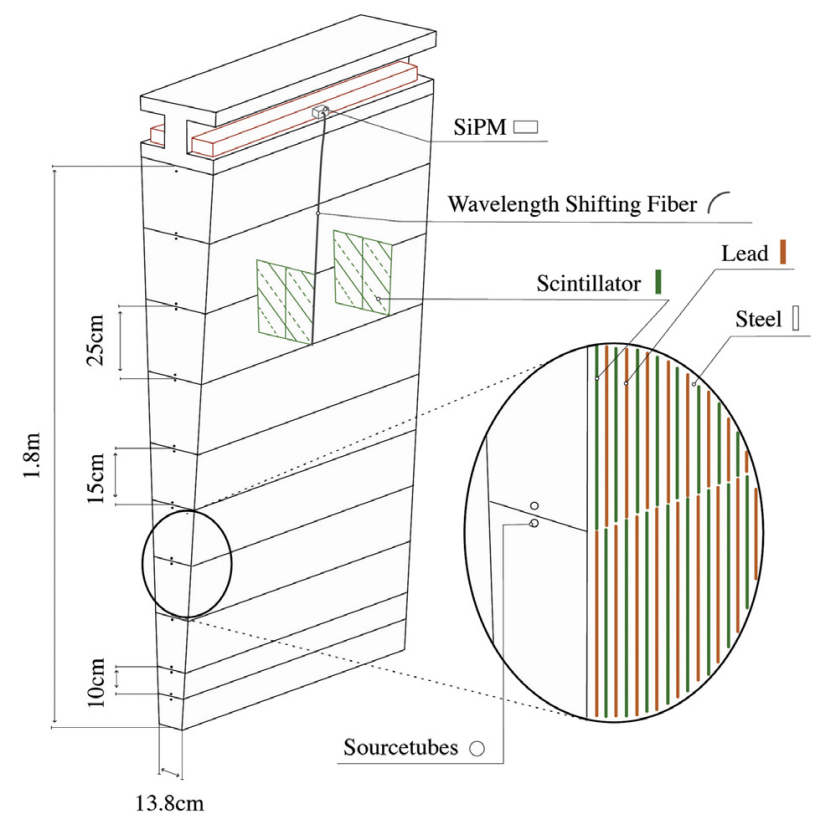

Fig. 4. Schematic of the HCal barrel Tile calorimeter module.

the inner tracker, but this has yet to be addressed. The out-of-time pileup could be added as a correction factor $(\times 1.5)$ but is not included here as HL-LHC aims at suppressing it to a large extent, thus should be doable at FCC-hh. A large impact of in time pile-up on the noise term is observed. Without any techniques for removal it increases from $0.3 \mathrm{GeV}$ to $4.4 \mathrm{GeV}$ for $\langle\mu\rangle=1000$. As shown in Fig. 3 it severely degrades $m_{\gamma \gamma}$ resolution. The Higgs self-coupling precision in the $H H \rightarrow b \bar{b} \gamma \gamma$ channel would degrade from 4 to $5 \%$ only by considering the worsening of the photon resolution. More elaborate techniques are needed to recover performance such as tracking and timing information, as well as improving clustering.

\section{Tile calorimeters}

The design of FCC-hh tile calorimeter is in appearance very similar to ATLAS [9]. Main differences are the partial substitution of steel absorber by lead. This has the advantage of decreasing the non-compensation by the suppression of the electromagnetic response. The current design of a module is sketched in Fig. 4. The nuclear interaction length of lead being smaller than steel, at $\eta=0$ a $0.4 \lambda$ depth reduction is

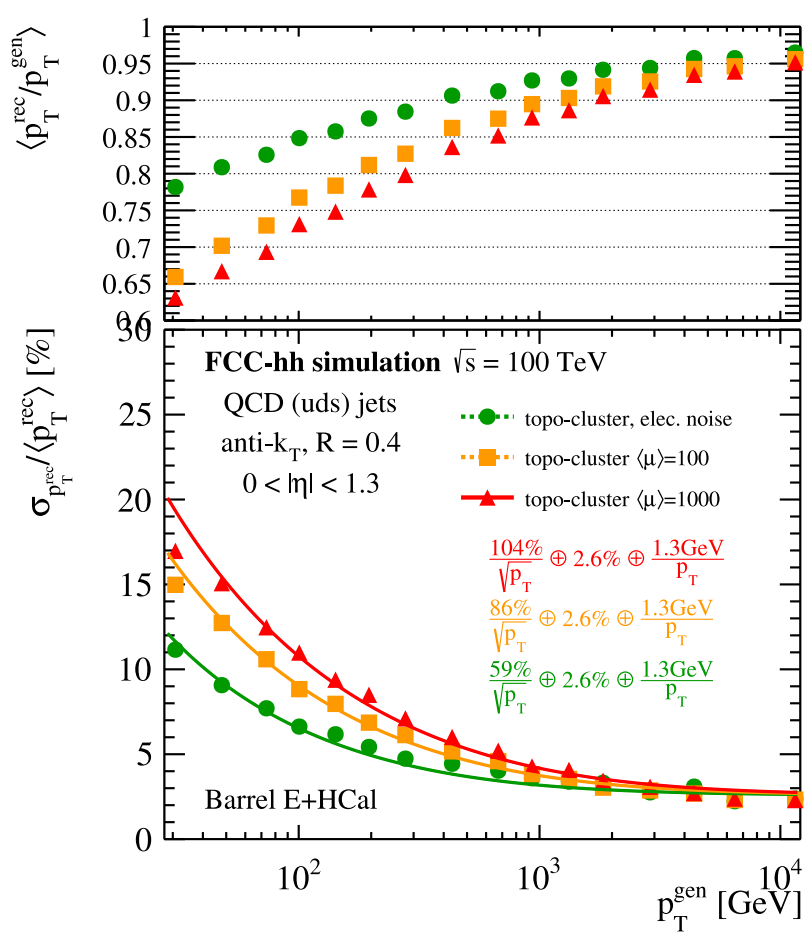

Fig. 5. Jet $p_{T}$ resolution after reconstruction using jet a clustering algorithm in three different noise scenarios without magnetic field.

conceded. One of the most interesting development is the substitution of photocathode by silicon photo-multipliers. As they are not sensitive to magnetic field, dedicated shielding is not needed. Unlike ATLAS, their small size allows to read out every tile longitudinally and in $\phi$, leading to a factor of ten increase in granularity $(\Delta \eta \times \Delta \phi=0.025 \times$ $0.025)$. The design considered for this study uses 10 longitudinal layers. Overall, mechanical structure and assembly of such calorimeter would be possible with present technologies.

Concerning the performances, the simulation of single pions with no noise in detector shows that the resolution goals for combined $\mathrm{ECal}+\mathrm{HCal}$ of $50 \% / \sqrt{E} \oplus 2.2 \%$ is achieved.

\section{Combined performances}

As shown in Fig. 5, excellent jet resolution up to $p_{T}=10 \mathrm{TeV}$ is observed without considering the magnetic field. As expected, the pileup has a large impact at low $p_{T}$, and it is crucial for low mass di-jet resonance searches, such as $H H \rightarrow b \bar{b} \gamma \gamma$ to mitigate it to a large extent. In principle this can be achieved using particle-flow where the charged hadron component of the pile-up is subtracted.

With the large number of pile-up interactions, a large amount of fake-jets from simple combinatorics could be reconstructed. In order to identify such jets both longitudinal and lateral segmentation are needed. Simplistic discriminating variables such as the energy deposited in each layer (a pileup-jet with $p_{T}>100 \mathrm{GeV}$ deposits twice more energy in the first two layers than a prompt jet), or the energy deposited in a given $d R$ of the jet axis (a prompt jet is more collimated) show possible handles, but could be further improved. Indeed, in practice, tracking, timing and particle-flow will help a lot.

Fig. 6 shows the jet mass for high $p_{T}$ objects in the standalone calorimeter and without magnetic field. It shows that with simplistic analysis some discrimination between $\mathrm{W}$ and $\mathrm{Z}$ boson can be observed and that very good separation with prompt QCD jets is achieved. The next steps would be to explore more sophisticated methods including particle-flow and machine learning. 


\section{FCC-hh Simulation}

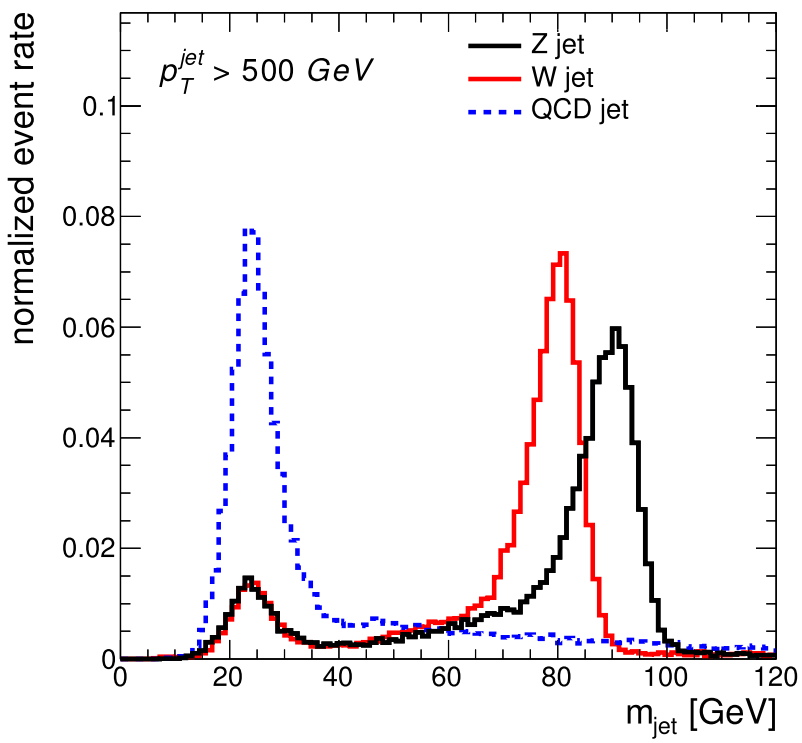

Fig. 6. Distribution of the jet mass for boosted W, Z and QCD jet with $p_{T}>500 \mathrm{GeV}$ without magnetic field.

\section{Summary}

A novel design of the electromagnetic and hadronic calorimeters for the FCC-hh experiments is presented. It is LAr-based for ECal and HCal in $1.4<|\eta|<6$ and for the ECal in $|\eta|<1.4$ and scintillator based for the HCal in $|\eta|<1.4$. In a no pile-up environment the resolution goals have been achieved: electrons/photons $(8 \% / \sqrt{E} \oplus 0.2 \%)$, pions $(50 \% \sqrt{E} \oplus 2.2 \%)$ and jets (without B-field $60 \% / \sqrt{E} \oplus 2.6 \%$ ). Pileup is a real challenge for FCC-hh environment and is valid for any studied detector option. Optimisation of the reconstruction procedures is necessary, and additional information such as tracking and timing needs to be taken into consideration to improve the performances. As expected, pile-up of $\langle\mu\rangle=1000$ is a hostile environment even for radiation-hard calorimetry as it strongly degrades the energy resolution. High longitudinal and lateral segmentation is suitable for pile-up jet identification, particle-flow algorithms and maintaining good angular and energy resolution. Dedicated studies are ongoing to improve the performance of the reconstruction methods.

\section{References}

[1] M. Benedikt, Study status and further plans, talk at FCC week https://indico.cern. ch/event/656491/timetable/, Amsterdam, 2018.

[2] FCC-hh design study https://fcc.web.cern.ch/Pages/fcc-hh.aspx/.

[3] FCCSW main page, http://fccsw.web.cern.ch/fccsw/.

[4] M.L. Mangano, Physics at the FCC-hh, a $100 \mathrm{TeV}$ pp collider, arXiv: 1710.06353.

[5] A. Zaborowska, FCC-hh and HE-LHC experiments and detectors overview, talk at the FCC week https://indico.cern.ch/event/656491/contributions/2915653/, Amsterdam, 2018

[6] G. Aad, et al., [ATLAS Collaboration], The ATLAS experiment at the CERN large hadron collider, JINST 3 (2008) S08003, http://dx.doi.org/10.1088/1748-0221/3/ 08/S08003.

[7] [ATLAS Collaboration], CERN-LHCC-96-41.

[8] Calorimeter Clustering Algorithms: Description and Performance. TL-LARG-PUB2008-002.

[9] [ATLAS Collaboration], CERN-LHCC-96-42. 